\title{
Similarity and Dissimilarity between Clinical and Laboratory Findings, Especially Anti-Thyrotropin Receptor Antibody in Ophthalmic Graves' Disease without Persistent Hyperthyroidism and Hyperthyroid Graves' Disease
}

\author{
Shinji KOSUgi, Daisuke INOUE, Hideo SugAWA, Tetsuya ENOMOTO, \\ TORU MORI AND HIROO IMURA
}

\begin{abstract}
Second Division, Department of Internal Medicine, and Clinical Molecular Biology, Kyoto University Faculty of Medicine, Kyoto 606, Japan
\end{abstract}

\begin{abstract}
The aim of this study was to investigate thyroid states, significance of anti-TSH receptor antibodies and the clinical courses of patients with euthyroid Graves' ophthalmopathy. The clinical and laboratory finding of 30 patients with euthyroid Graves' ophthalmopathy were briefly as follows: 1) normal sized thyroid or small goiter; 2) negative or weakly positive thyrotropin binding inhibitor immunoglobulin (TBII) ; 3) normal thyroid [99 mTc] pertechnetate uptake; and 4) frequent observations of low serum TSH values. Besides TBII, thyroid stimulating antibody (TSAb) was measured under low salt and isotonic conditions using FRTL-5 rat thyroid cells. Both TBII and TSAb titers were lower in euthyroid Graves' ophthalmopathy than in hyperthyroid Graves' disease. Serum TSH levels frequently became low in patients considered as euthyroid upon the first examination as well as in Graves' patients in remission, reflecting preceding or mild hyperthyroidism. In follow-up studies, these patients with mildly elevated thyroid hormone levels and low TSH levels seldom reached a state of persistent hyperthyroidism, when TBII was negative or only weakly positive.
\end{abstract}

As well known, Graves' ophthalmopathy is not always associated with hyperthyroidism. Such a condition has been reported under various terms and its' etiological similarity or dissimilarity to that of hyperthyroid Graves' disease has been argued. In the present paper we tentatively desig-

Received September 13, 1989

Correspondence and reprint request: SHINJI KOSUGI, M. D., Second Division, Department of Medicine, Kyoto University School of Medicine, 54 Shogoinkawaharacho, Sakyoku, Kyoto 606, Japan. nated the condition as 'euthyroid Graves' ophthalmopathy'.

Solomon et al. (1977) insisted that Graves' ophthalmopathy is a disease entity independent of Graves' disease or Hashimoto's thyroiditis, although these diseases are frequently associated. In contrast, some investigators considered that the etiologies of both diseases are essentially identical, and Kasagi et al. $(1986 ; 1987)$ reported almost consistent detectability of thyroid stimulating antibody (TSAb) in patients' sera. 
As for the lack of hyperthyroidism, the thyroid gland of patients with euthyroid ophthalmopathy may show various degrees of unresponsiveness to TSH and anti-TSH receptor antibodies presumably owing to inflammatory changes (Kasagi et al., 1986, 1987 ; Teng et al., 1977). An alternative possible reason for this unresponsiveness is the qualitative difference in anti-TSH receptor antibodies in the two diseases (Kasagi et al., 1987). Further, by means of histocompatibility lymphocytic antigen (HLA) typing we have recently observed an apparent genetic differences between euthyroid Graves' ophthalmopathy and hyperthyroid Graves' (Inoue et al., unpublished observation). To further elucidate the pathogenesis, we studied patients with euthyroid Graves' ophthalmopathy in terms of their thyroid states, anti-TSH receptor antibodies and their clinical courses.

\section{Patients and Methods}

\section{Patients}

Serum samples were obtained from 33 normal subjects, 26 untreated hyperthyroid Graves' patients and 30 euthyroid Graves' ophthalmopathy patients.

The diagnosis of hyperthyroid Graves' disease was based on clinical signs, symptoms and laboratory findings including diffuse goiter, elevated serum thyroid hormone concentrations together with suppressed TSH, and an increased thyroidal uptake of $[99 \mathrm{~m}-\mathrm{Tc}]$ pertechnetate at $20 \mathrm{~min}$.

The definition of euthyroid Graves' ophthalmopathy employed was as follows : 1) the existence of Graves' ophthalmopathy; bilateral exophthalmos exceeding $16 \mathrm{~mm}$ and swelling of extraocular musculi confirmed by computed tomography (coronal section); 2) no previous history of treatment for hyperthyroidism as well as the lack of laboratory findings of persistent thyrotoxicosis without treatment; 3) TSH concentration lower than $0.3 \mu \mathrm{U} / \mathrm{ml}$ did not last more than 6 months without treatment. None of the 30 patients presented developed persistent hyperthyroidism during at least 2 years of observation.

\section{Laboratory examination}

Serum TSH was measured by a highly sensitive enzyme-immunoassay provided by Daiichi Radioisotope Laboratories (Tokyo, Japan) with a minimal detectable quantity of $0.005 \mu \mathrm{U} / \mathrm{ml}$, which can well differentiate low and suppressed TSH values from low normal TSH concentrations. TRH test was performed with a $500 \mu \mathrm{g}$ intravenous bolus injection of TRH tartrate (Hirutonin, Takeda, Osaka, Japan). Serum TSH concentrations before and 30,60 , 90 and 120 min after the in jection were measured. Normal response was defined as when the peak value exceeded $6 \mu \mathrm{U} / \mathrm{ml}$. Serum $T_{4}$ and $T_{3}$ were measured with commercially available RIA kits (Daiichi Radioisotope for $\mathrm{T}_{4}$, Dainabot (Tokyo, Japan) for T3). Serum free $T_{4}$ was measured with a kit from Amersham International plc. using a T4 conjugate (Buckinghamshire, England). Anti-thyroid microsomal antibody (anti-M) was assayed with a passive agglutination kit (Fujirebio, Tokyo, Japan). Thyroid [ $99 \mathrm{~m}-\mathrm{Tc}]$ pertechnetate uptake was measured $20 \mathrm{~min}$ after the intravenous injection of a $74 \mathrm{MBq}$ dose. The normal range is from 0.4 to $2.5 \%$. $\mathrm{T}_{3}$ Suppression test was performed following 7-day administration of $l-T_{3}$ at a daily dose of $75 \mu \mathrm{g}$. Positive suppression was defined when post $\mathrm{T}_{3}$ thyroid ${ }^{99} \mathrm{~m} \mathrm{TcO}_{4}^{-}$uptake was less than $1 \%$ and less than a half of the initial value. TBII (thyrotropin binding inhibitor immunoglobulin) was assayed with a Smith's kit (Baxter-Travenol Inc., Tokyo, Japan) with correction by individual nonspecific binding (Akamizu et al., 1987). The normal range is from -10 to $+10 \%$.

\section{TSAb assay}

TSAb (thyroid stimulating antibody) was assessed by CAMP production in FRTL-5 cells under both low and high salt incubation conditions.

FRTL-5 rat thyroid cells kindly provided by Dr. Kohn (NIH, Bethesda, MD) were cultured as described by Vitti et al. (1983) with minor modifications. The cells were seeded in 24-well plates (Corning, N. Y) as evenly as possible to make $5 \times 10^{4}$ cells $/$ well. The cells were cultured in $6 \mathrm{H}$ medium for 7 days in $95 \%$ air $-5 \% \mathrm{CO}_{2}$ at $37^{\circ} \mathrm{C}$ and the medium was replaced with fresh medium on day 4 . The $6 \mathrm{H}$ medium comprised Coon's modified Ham's F-12 medium 
containing 5\% calf serum (Flow, North Ryde, Australia), $0.1 \mathrm{mM}$ non-essential amino acids (Gibco, NY), 0.03\% glutamine and 6 hormones, i. e., $300 \mu \mathrm{U} / \mathrm{ml}$ bovine TSH (bTSH; Sigma Chemical Co., St. Louis, MO), $10 \mu \mathrm{g} / \mathrm{ml}$ insulin (Sigma), $10^{-9} \mathrm{M}$ hydrocortisone (Sigma), $5 \mu \mathrm{g} /$ $\mathrm{ml}$ human transferrin (Sigma), $10 \mathrm{ng} / \mathrm{ml}$ somatostatin (Peptide Institute Inc., Osaka, Japan) and $2 \mu \mathrm{g} / \mathrm{ml}$ glycyl-L-histidyl-L-lysine acetate (Sigma). When the cells reached confluence, the medium was replaced with $5 \mathrm{H}$ medium $(6 \mathrm{H}$ medium without bTSH) and the cells were cultured for another 7 days. Again at day 4 the $5 \mathrm{H}$ medium was replaced with fresh medium.

Preparation of immunoglobulin (Ig) from sera was performed by essentially the same procedures as described by Kasagi et al. (1986, 1987). $0.4 \mathrm{ml}$ aliquots of serum samples were mixed with $1.2 \mathrm{ml} \mathrm{20 \%} \mathrm{(wt/vol)} \mathrm{polyethyleneglycol}$ (mol wt 4000) and centrifuged at $2000 \times \mathrm{g}$ for $30 \mathrm{~min}$. The resulting pellet was dissolved in either $0.6 \mathrm{ml}$ of Hank's solution (high salt) or $\mathrm{NaCl}$-free low salt modified Hank's solution (low salt). Both solutions contained $1.5 \%(\mathrm{wt} /$ vol) BSA, $20 \mathrm{mM}$ N-2-hydroxyethylpiperazine$\mathrm{N}$ '-2-ethanesulfonic acid (HEPES) adjusted to $\mathrm{pH}$ 7.4 with $1 \mathrm{M} \mathrm{NaOH}$, and $0.5 \mathrm{mM} 3$-isobutyl-1methylxanthine.

After aspirating the $5 \mathrm{H}$ medium, FRTL-5 cells in 24-well plates were incubated with 0.3 $\mathrm{ml}$ of Ig preparations for $120 \mathrm{~min}$ at $37^{\circ} \mathrm{C}$. Following incubation, the media were collected to measure cAMP by RIA in duplicate with a Yamasa cAMP assay kit (Choshi, Japan). Intracellular cAMP was extracted with $0.1 \mathrm{M} \mathrm{HCl}$ and was also applied to the cAMP assay. $\mathrm{TSAb}$ activity of individual Ig was expressed as a percent difference from cAMP generated from that of normal Ig samples. The normal range determined in 33 normal subjects was from 50 to $150 \%$ (mean $\pm 2 \mathrm{SD})$.

\section{Results}

\section{Laboratory data}

Laboratory data from the first examination of the euthyroid Graves' ophthalmopathy patients studied are shown in Fig. 1. A small goiter (less than $5 \mathrm{~cm}$ in diameter) or a normal sized thyroid was found by palpation and ultrasound echography in each of the 30 patients. The serum TSH concentration in these patients was often lower than the lower normal limit, even though their thyroid hormone was essentially within the normal range. Three patients with normal TSH upon the first examination transiently had low TSH afterwards. Reference case 1 whose data are shown by $D \leftarrow$ in Fig. 1. will be detailed later. Three such patients had a higher thyroid hormone concentration $\left(T_{4}, T_{3}\right.$ or free $\left.T_{4}\right)$ but had normal TSH and were judged as euthyroid. As for the interrelationship between serum TSH and thyroid hormone, TSH vs. $\mathrm{T}_{4}$ and TSH vs. free $\mathrm{T} 4$ showed significant inverse correlations $(r=-0.578$ and -0.601 , respectively; calculated from log transformed TSH values). In most (93\%) of the patients with euthyroid Graves' ophthalmopathy thyroid ${ }_{99} \mathrm{Tc}_{4}{ }^{-}$uptake remained within the normal range. One case had higher uptake, and one lower uptake. The former was not associated with any abnormal values in blood testing and the latter had increased $\mathrm{T}_{4}$ with normal TSH, $\mathrm{T}_{3}$ and free $\mathrm{T}_{4}$. Anti-M titers were high in some patients but detectable in only $34 \%$ of the patients. Three of the low TSH patients were positive for the antibody, but 4 others were negative.

\section{TBII and TSAb activity}

TBII activity was mostly negative or weakly positive (lower than 17\%) in these patients. Only $3(10 \%)$ of the 30 patients studied were positive for TBII and 1 of them had low TSH 2 years after the initial examination. TSAb under low salt conditions was positive in $22 \%$ of the euthyroid Graves' ophthalmopathy patients and the highest value among them was $334 \%$. Four of 7 low TSH subjects were found to have positive TSAb also, but the subject with the highest TSAb had normal TSH $(0.8 \mu \mathrm{U} /$ $\mathrm{ml})$.

As shown in Fig. 2, excellent correlations 
Fig. 1 A

Fig. 1. Serum TSH, $\mathrm{T}_{4}, \mathrm{~T}_{3}$ and free $\mathrm{T}_{4}$ concentrations and the thyroid ${ }^{99} \mathrm{~m} \mathrm{TcO}_{4}^{-}$uptake (A) and antimicrosomal antibody, TBII and TSAb (low salt condition) (B) on the first examination of patients with euthyroid Graves' ophthalmopathy.

Shaded areas are the normal ranges. Closed circles represent patients

I. whose TSH concentrations were more than 0.3 $\mu_{3}^{\prime} \mathrm{U} / \mathrm{ml}$, and open circles, less than that. Semiclosed circles represent patients whose TSH concentrations were within the normal range at the beginning and transiently decreased less than $0.3 \mu \mathrm{U} / \mathrm{ml}$ during obsesva-
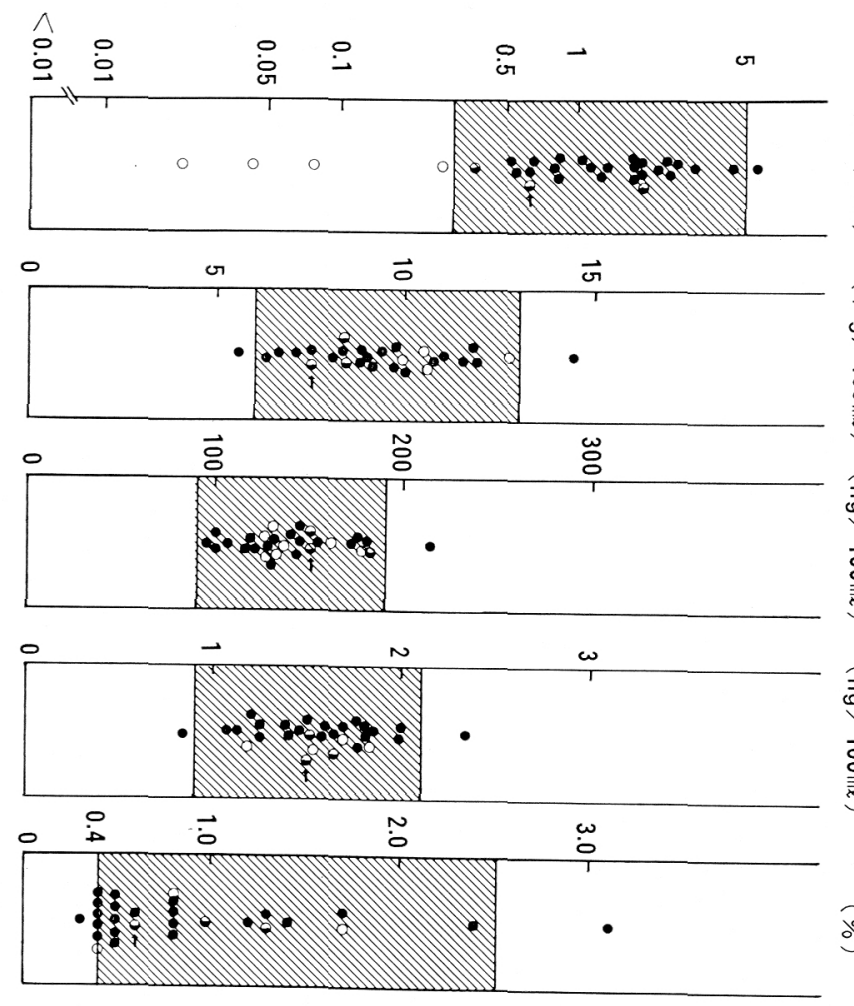

Fig. 1 B tion.

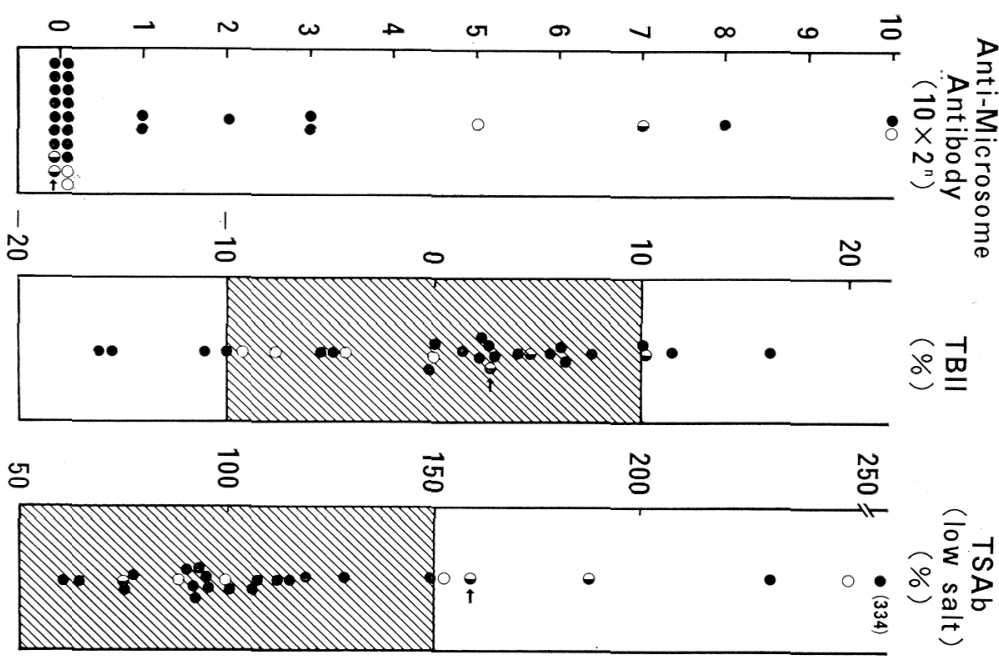

were observed between TBII and TSAb under both low and high salt conditions among euthyroid Graves' ophthalmopathy and hyperthyroid Graves' patients. Under the low salt conditions $96 \%$ of the hyperthyroid Grave's patients gave positive results for $\mathrm{TSAb}$ and all of them were positive for TBII. Furthermore, even in cases with equipotent TBII, TSAb under low salt conditions was lower in euthyroid Graves' ophthalmopathy patients. Though there were several overlapping cases in each category, the distribution of euthyroid Graves' ophthalmopathy was apparently different from that of hyperthyroid Graves'. TSAb under high salt conditions did not alter the above tendency (Fig. 2 B) and showed a good correlation with that under low salt conditions ( $\mathrm{r}$ $=0.763, \quad n=53$, calculated from log transformed data including both groups).

To illustrate the clinical significance of TBII and TSAb, TRH test and $\mathrm{T}_{3}$-suppression test results are shown in Table 1. These 


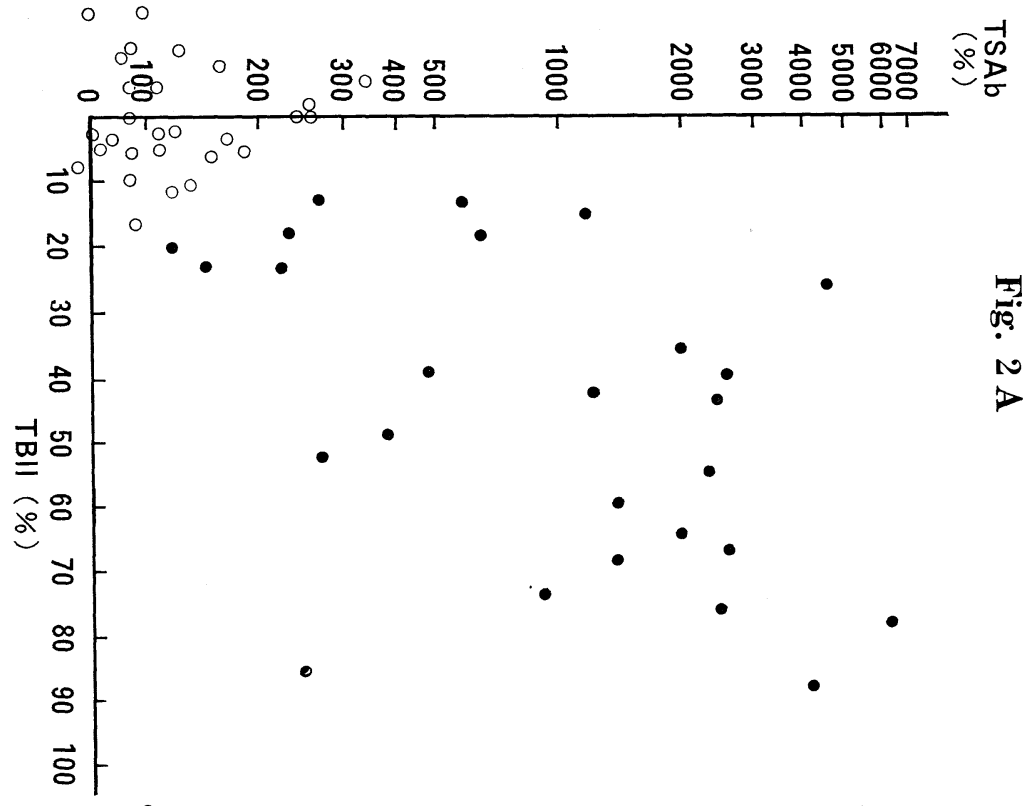

tests were performed independently on a limited number of patients (19 and 18 cases, respectively). Impaired or blunted TSH response to TRH was observed in 3 of 5 low TSH subjects and none of 14 normal TSH subjects, 2 out of 5 TBII positive and 1 of 12 negative subjects, and 2 out of 5 TSAb positive and 1 of 14 negative subjects. Negative $\mathrm{T}_{3}$-suppressibility was encountered in 3 of 5 low serum

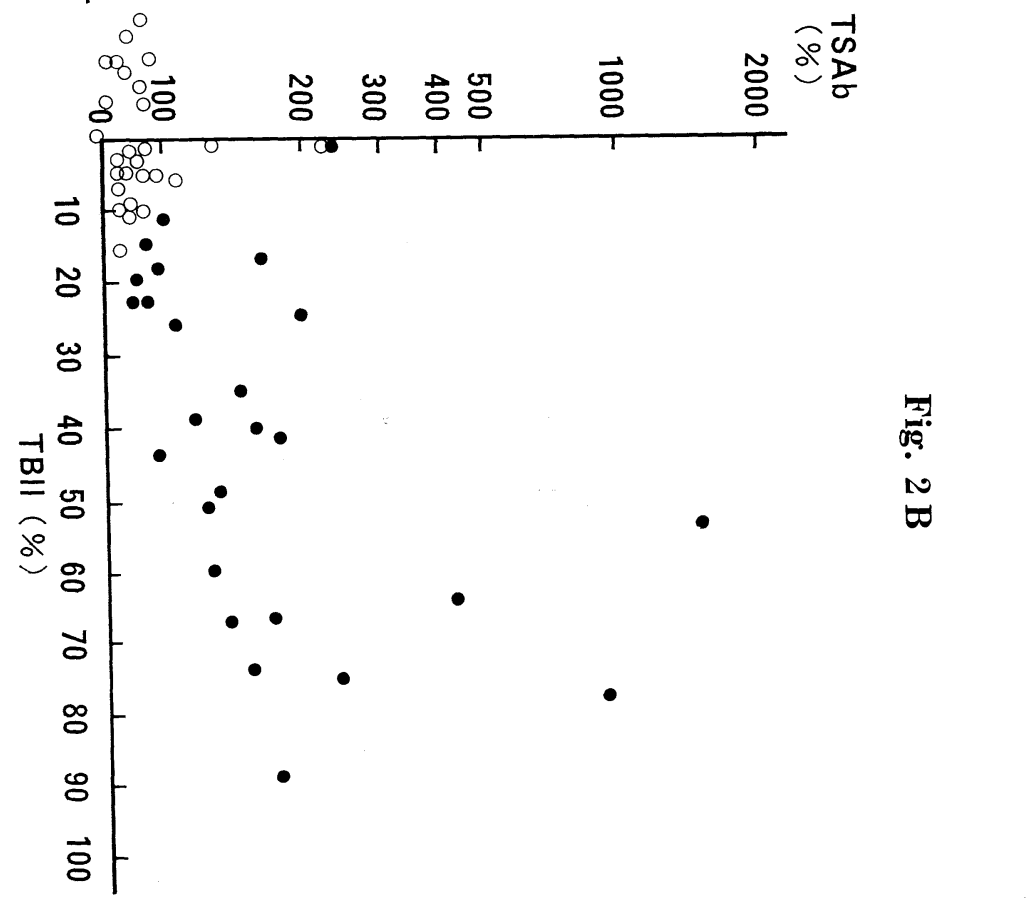

Fig. 2. Correlation between TBII and TSAb under low salt (A) and high salt (B) conditions in patients with euthyroid Graves' ophthalmopathy and hyperthyroid Graves' disease. Open circles are euthroid Graves' ophthalmopathy and closed circles are untreated hyperthyroid Graves'. Extracellular amounts of cAMP were assessed for TSAb under low salt conditions and a total (extracellular and intracellular) cAMP was assessed under high salt conditions. The correlation efficiencies for A and B were $\mathrm{r}=0.745(\mathrm{n}=53)$ and $\mathrm{r}=0.514(\mathrm{n}=53)$, respectively (calculated from log transformed TSAb values). TSH subjects, 3 of 13 normal TSH subjects, 2 of 6 TBII positive and 4 of 12 negative subjects, and 2 of 5 TSAb positive and 4 of 13 negative subjects. Further, normal TRH test results and positive $\mathrm{T}_{3}$-suppression were observed in subjects with low TSH, positive TBII and/or TSAb.

Reflecting the instability of the thyroid states in these patients, none of these parameters appeared to clearly indicate that they were Graves' disease. When at least one of these parameters is abnormal, the patient may be taken as having Graves' disease and this was so in 17 of 
Table 1. TRH test results and $\mathrm{T}_{3}$-suppressibility of patients with euthyroid Graves' ophthalmopathy in relation to TSH, TBII and TSAb

\begin{tabular}{|c|c|c|c|c|c|c|}
\hline & \multicolumn{2}{|c|}{$\operatorname{serum} \operatorname{TSH}(\mu \mathrm{U} / \mathrm{ml})$} & \multicolumn{2}{|c|}{ TBII } & \multicolumn{2}{|c|}{$\mathrm{TSAb}^{\mathrm{a}}$} \\
\hline & $<0.3$ & $\geqq 0.3$ & + & - & + & - \\
\hline \multicolumn{7}{|l|}{ TRH test } \\
\hline normal & 2 & 14 & 5 & 11 & 3 & 13 \\
\hline impaired & 3 & 0 & 2 & 1 & 2 & 1 \\
\hline \multicolumn{7}{|c|}{$T_{3}$-suppression } \\
\hline positive & 2 & 10 & 4 & 8 & 3 & 7 \\
\hline negative & 3 & 3 & 2 & 4 & 2 & 4 \\
\hline
\end{tabular}

$\mathrm{a}:$ TSAb activity measured under low salt conditions.

Serum TSH, TBII and TSAb were measured repeatedly during the observation period, and abnormal results even on one occasion are shown as abnormal. Therefore, the numbers with abnormal TBII and TSAb are different from those in Fig. 1B which shows activity on the first examination.

23 well studied patients $(74 \%)$. In four of the remaining $(17 \%)$ these parameters were normal but were positive only for anti-M and should not to be taken as indicating Graves' disease. Furthermore, in 2 patients. all these results were negative. As to the criteria of euthyroid Graves' ophthalmopathy applied to the present study, most of the patients $(91 \%)$ were found to be dysthyroidal in nature but could not be definitely classified as Graves' disease. Therefore euthyroid ophthalmopathy may be a more suitable name, but euthyroid Graves' ophthalmopathy was used in the text for convenience.

\section{Specific cases}

Several ophthalmopathy cases are presented to help provide an understanding of the pathophysiology of the disease. Case 1 was a 21-year-old female with bilateral exophthalmos (right: $16.0 \mathrm{~mm}$, left : 17.0 $\mathrm{mm}$ ) and normal sized thyroid (transverse diameter: $3.0 \mathrm{~cm}$ ). Upon initial examination, $\mathrm{T}_{4}, \mathrm{~T}_{3}$, free $\mathrm{T}_{4}, \mathrm{TSH}$ and thyroid ${ }^{99} \mathrm{TcO}_{4}^{-}$uptake $(0.6 \%)$ were all within the normal range, and TBII was negative. From these observations the patient was diagnosed as having euthyroid Graves' ophthalmopathy. No medication was prescribed. As shown in Fig. 3, apparent TSH suppression during the 15 month observation period was repeatedly seen in association with a slight increase in thyroid hormones. The thyrotoxic state did not last long and the possibibility of tissue destruction was ruled out by normal thyroid ${ }^{99} \mathrm{~m} \mathrm{TcO}_{4}{ }^{-}$uptake. Interestingly, in the first episode of hyperthyroidism, TBII was also increased slightly but significantly. A negative TBII result was obtained 3 months later, and the second episode was not associated with any TBII increase.

The observations in this case clearly indicate fluctuations in thyroid states in euthyroid Graves' ophthalmopathy patients. The following 2 cases treated with antithyroid drug were not included among euthyroid Graves' ophthalmopathy cases but showed interesting clinical courses.

Case 2 (Fig. 4) was a 47-year-old female patient. Exophthalmos was measured as $19.0 \mathrm{~mm}$ (right) and $16.5 \mathrm{~mm}$ (left) and the goiter size was $4.2 \mathrm{~cm}$ in transverse diameter. Initially, $\mathrm{T}_{3}$ and $\mathrm{T}_{4}$ were in the upper normal ranges and TBII was negative, but TSH was not detectable. Thyroid ${ }^{99} \mathrm{TcO}_{4}^{-}$uptake was within the normal range $(1.3 \%)$ but was not suppressed by $\mathrm{T}_{3}$. Medication was not administered to this patient either. After 4 months, $T_{3}$ and $\Gamma_{4}$ further increased beyond the upper limits of the normal range and TBII became weakly positive. Then, a low dose (10 mg/day) of MMI (2-methylmercaptoimidazole) was prescribed. $\mathrm{T}_{3}, \mathrm{~T}_{4}$ and TBII returned to within the normal range after only 1 month of medication. TSH also became detectable and shortly returned to the normal range. After 12 months from the beginning of medication, positive T3-suppression was achieved and the medication was stopped. The patient has been 
Fig. 3

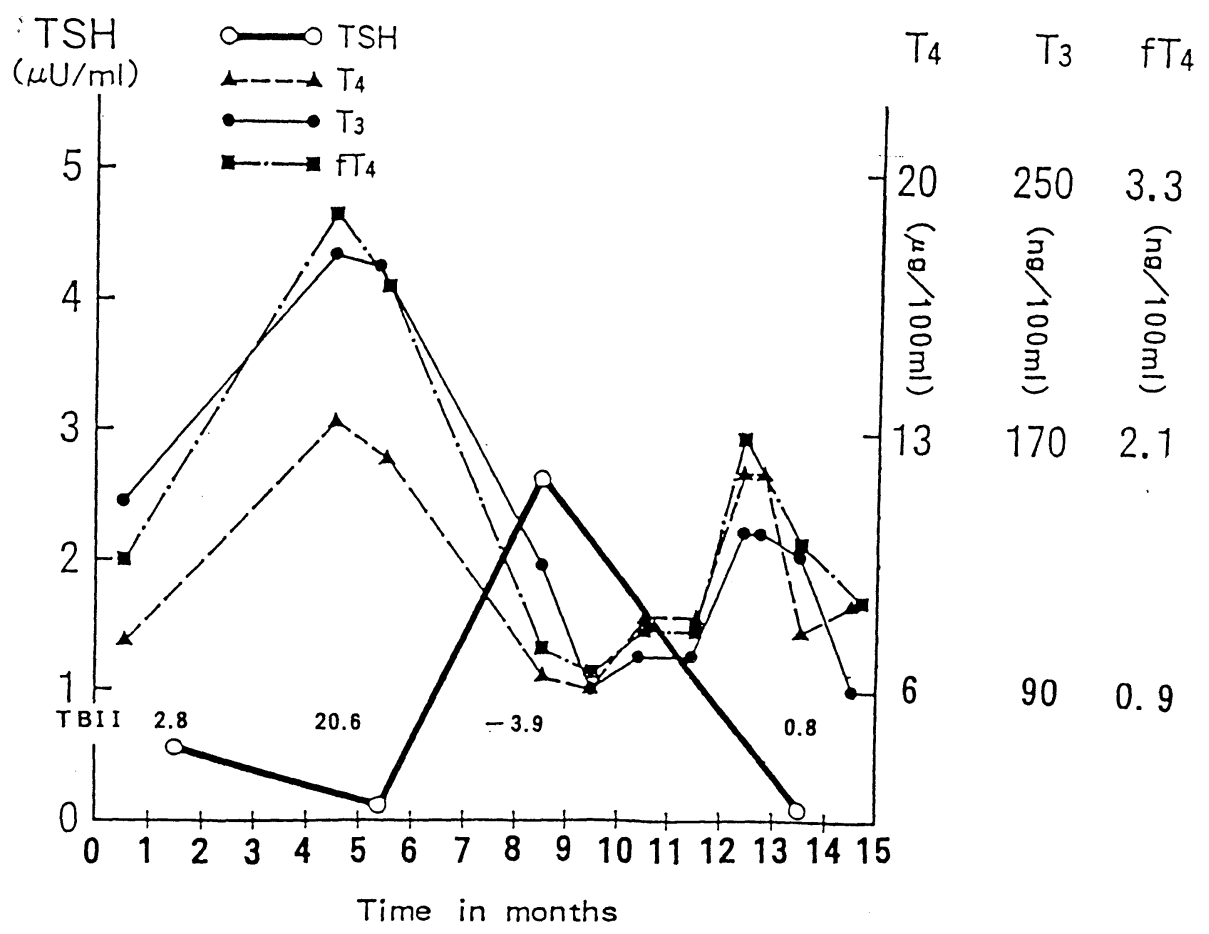

Fig. 3. Changes in thyroid functions and TBII in a patient (case 1).

Fig. 4

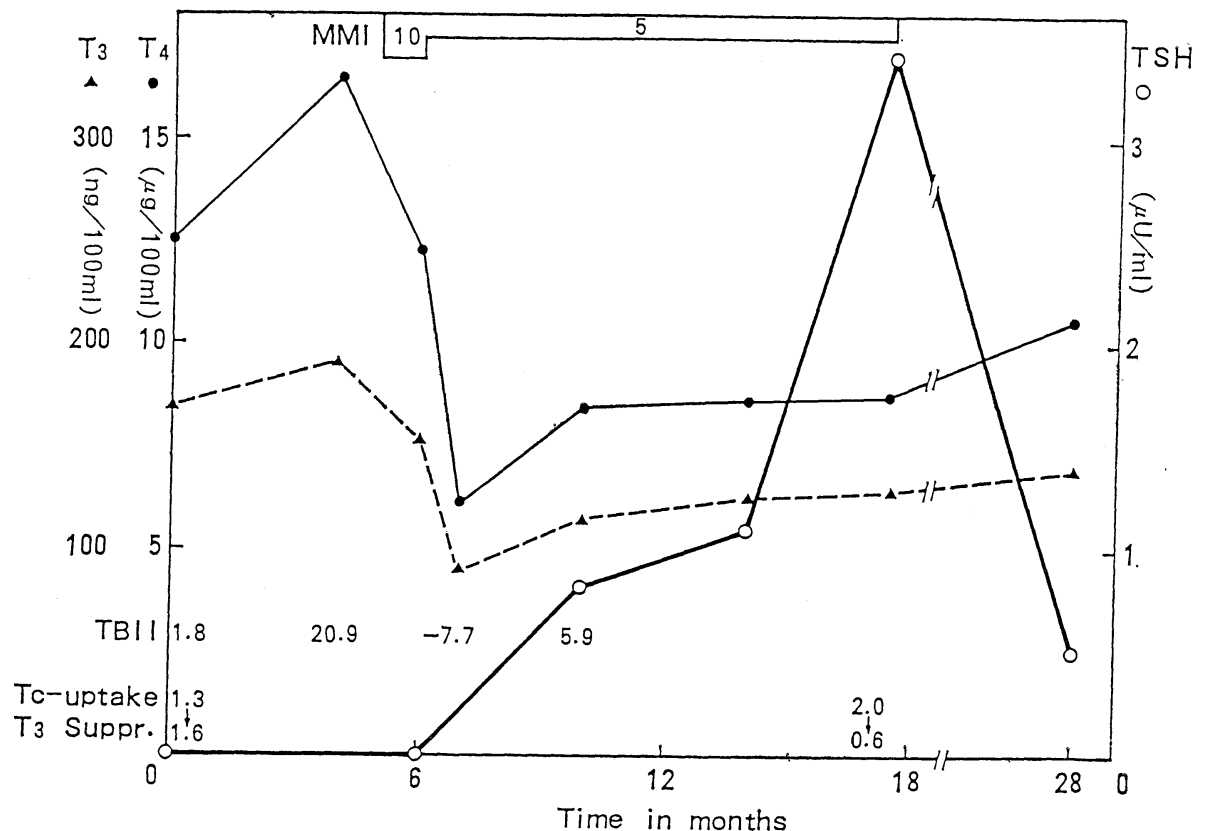

Fig. 4. Changes in thyroid functions and TBII in a Graves' patient (case 2). 
in a euthyroid state since then.

Case 3 (Fig. 5) was a 32-year-old male patient. Bilateral exophthalmos (right: $16.0 \mathrm{~mm}$, left : $17.0 \mathrm{~mm}$ ) and a normal sized thyroid (transverse diameter, $3.0 \mathrm{~cm}$ ) were present. During the initial observation period, $\mathrm{T}_{3}$ and $\mathrm{T}_{4}$ were high and TSH was undetectable, but ${ }^{99} \mathrm{mcO}_{4}{ }^{-}$ uptake was low, a1though not suppressed by $\mathrm{T}_{3}$. TBII was negative. The patient did not complain of any thyrotoxic signs and was followed without medication. $\mathrm{T}_{3}$ and $\mathrm{T}_{4}$ decreased gradually to the upper normal levels, and TSH became detectable within 2 months.

However, 3 months thereafter, $T_{3}$ and $T_{4}$ slightly increased again and TSH decreased. A low dose (15 mg/day) of MMI was given. After only 7 months of this low dose MMI treatment, TSH returned to the normal range, $T_{3}$ suppresstion test turned out to be positive, and medication was stopped. Thyroid functions have been maintained within the normal range since then. TBII was negative throughout the observation period.

Case 4 (Fig. 6) was a 25-year-old female
Fig. 5

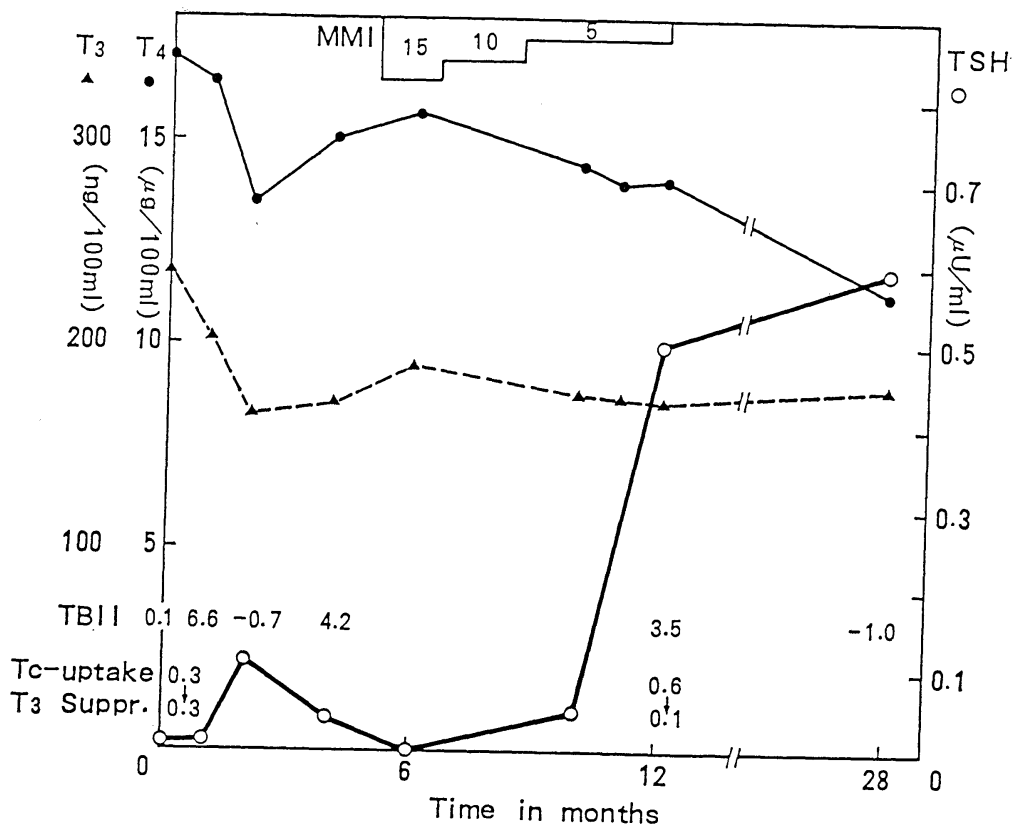

Fig. 5. Changes in thyroid functions and TBII in a Graves' patient (case 3).

Fig. 6

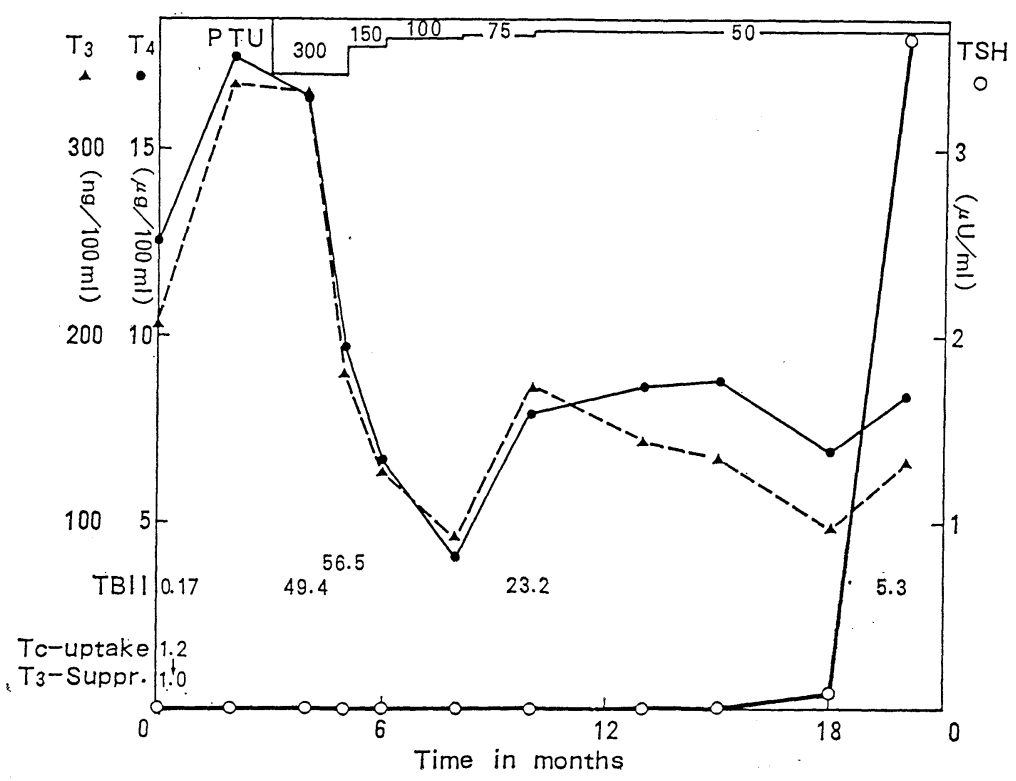

Fig. 6. Changes in thyroid functions and TBII in a patient with early Graves' (case 4). 
patient with exophthalmos (right: $16.0 \mathrm{~mm}$, left : $17.0 \mathrm{~mm}$ ) and a small diffuse goiter (transverse diameter, $4.5 \mathrm{~cm}$ ). The initial findings were quite similar to those of cases 1,2 and 3 ; a mild increase in $\mathrm{T}_{3}$ and $\mathrm{T}_{4}$ with suppression of $\mathrm{TSH}$, normal but not $\mathrm{T}_{3}$-suppressible ${ }^{99} \mathrm{~m} \mathrm{TcO}_{4}^{-}$ uptake, and negative TBII. Thyroid functions increased further and TBII became moderately positive during 4 months without medication after the initial observation. The hyperthyroidism persisted and was controlled by PTU (propylthiouracil) with a usual clinical course of hyperthyroid Graves' disease. TBII remained positive 7 months after treatment and turned out to be negative after 17 months. This patient was retrospectively diagnosed as having early Graves' disease.

\section{Discussion}

From the present study of 30 patients, euthyroid Graves' ophthalmopathy was characterized briefly as follows : 1) small goiter or normal sized thyroid; 2) normal thyroid ${ }_{99} \mathrm{TcO}_{4}-$ uptake ; 3) frequent low or suppressed TSH concentration; and 4) negative or weakly positive for TBII and TSAb. In this study we have used the nomenclature of euthyroid Graves' ophthalmopathy. However, in a restricted meaning, those who had lower TSH levels could not be considered to be euthyroid. We defined euthyroid Graves' ophthalmopathy as Graves' ophthalmopathy without persistent hyperthyroidism, and that is what we would like to describe here. Most of the patients reported previously who were fallen under the nomenclatures of euthyroid Graves', and dysthyroidal, endocrinological or autoimmune ophthalmopathy and so on, appear to be included in the present category. Classical TSH assays could not differentiate between low TSH values and low normal TSH values. These patients, previously reported, were thought to have a normal thyroid hormone level but they might have had low TSH if their TSH concentration had been measured by high sensitive TSH assays.

As proposed by Solomon et al. (1977), if the ophthalmopathy has an etiology quite different from hyperthyroid Graves', the absence of an association of persistent hyperthyroidism in these patients can be well understood. Evidence of autoimmune mechanisms specific to the eye has been reported (Etienne et al., 1976; Munro et al., 1973). However, the association of minimal or mild thyroidal abnormality with euthyroid ophthalmopathy is also very commonly seen, and abnormal TSH response to TRH or negative $\mathrm{T}_{3}$-suppression of thyroid radioiodide uptake has been reported (Gorman, 1978, Tamai et al., 1980). Here, we observed lower serum TSH in 7 out of 30 patients studied. Interestingly, these 7 patients who had lower serum TSH were found to have a nomal value during the observation period without any treatment. Similarly, Tamai et al., (1980) reported sequential changes in both TRH test results and T3-suppressibility in their patients. As for anti-thyroid microsomal antibody, a third of the patients in this series had an increase and 7 of the 10 positive cases were not associated with low serum TSH. From these observations. it is likely that at least some of the euthyroid Graves' ophthalmopathy patients have abnormal findings compatible with hyperthyroid Grave's or autoimmune thyroid disorders. The underlying problems are why these patients do not develop hyperthyroid Graves'. One possible explanation for the former is impaired responsiveness of the thyroid gland to TSH or anti-TSH receptor antibodies, probably due to inflammary tissue damages (Kasagi et al., 1986, 1987 ; Teng et al., 1977). The thyroid histological findings of these patients were reported to show features of Hashimoto's thyroiditis (Tamai et al., 1980). In contrast to these reports, 
we previously observed that euthyroid Graves' ophthalmopathy patients responded well to exogenous TSH administration. Further, when the serum $T_{3}$ concentration was measured after TRH injection, all of the normal TSH response cases studied showed a significant increase in serum $\mathrm{T}_{3}$ concomitantly. In addition, histology samples obtained by Silverman needle biopsy revealed only slight inflammatory or degenerative changes in 2 of 9 patients studied (unpublished observations).

In the present series, we observed no remarkable enlargement of the thyroid gland and almost normal ${ }^{99 \mathrm{~m} T c}$ pertechnetate uptake together with normal circulating thyroid hormones. The levels of anti-TSH receptor antibodies in these patients are also of interest. Most previous reports have not shown high detectability or high activity in patients with euthyroid ophthalmopathy (Bidey et al., 1983; Zakarija et al., 1980). In the present study, TBII in these patients was mostly negative or weakly positive. Kasagi et al. $(1986,1987)$ also observed similar results for TBII, but insisted that the very high detectability and high activity of TSAb in their patients was comparable with untreated hyperthyroid Graves' patients. They applied a low salt hypotonic incubation system for TSAb assay to improve the assay sensitivity. This non-physiological condition was found to induce certain kinds of damage in cultured thyroid cells, but at the same time to augment cAMP production further to increase sensitivity, especially to TSAb of Graves' Ig (Kosugi et al., 1989). In contrast to the results reported by Kasagi et al. (1986, 1987), we found that TSAb activity measured under low salt condition in these euthyroid Graves' ophthalmopathy patients was either negative $(78 \%)$ or only weakly positive $(22 \%)$ in contrast to high TSAb activity in peristent hyperthyroid Graves' patients (96\% positive). If Kasagi's observations were the case, the difference between euthyroid Graves' oph- thalmopathy and hyperthyroid Grave' may result from lower TBII in the former. Only with the coexistence of TBII, TSAb measured under low salt conditions may be stimulatory to the thyroid. The assumption that low salt conditions may affect the thyroid cell membrane to expose TSAb binding sites which can also be exposed by the binding of TBII during high salt conditions is certainly of interest. This was the reason why we measured TSAb under both low and high salt conditions, and the results revealed no essential changes in either condition. From these observations, we concluded that patients with euthyroid Graves' ophthalmopathy have a negative or low level of anti-TSH receptor antibodies.

It was reported previously that a close correlation was found between TBII and thyroid ${ }^{99 \mathrm{~m} T \mathrm{c}}$ pertechnetate uptake, goiter size and grade of epithelial hyperplasia in Graves' patients (Akamizu et al., 1984). A weak association of TBII and TSAb with euthyroid Graves' ophthalmopathy is consistent with their smaller goiter size and normal thyroid uptake. The reason why serum TSH in some of these patients was suppressed remains to be explained. At present, the serum TSH concentration, measured by a highly sensitive assay, is believed to be the most sensitive indicator of thyroid dysfunction (Glee \& Hay, 1987; Mori et al., 1987). Low TSH values reflect the presence of subclinical hyperthyroidism at that time or some time shorty before the measurement. As shown in Fig. 2, those who exhibited suppressed TSH were not always associated with positive TBII or TSAb, and most of them were even negative for the antibodies. However, noteworthy that in cases 1,2 and 4 a concomitant increase in circulating thyroid hormone and TBII was observed. Additionally, a persistent and marked increase in TBII in case 4 resulted in the development of hyperthyroid Graves'. Thus, antiTSH receptor antibody, especially TBII, 
appeared to play a role in the development and maintenance of hyperthyroidism. Some patients (like cases 2 and 3 ) who were given small doses of antithyroid drugs for a short period, and recovered to euthyroid rapidly and remained euthyroid after cessation of the treatment, might have returned to a euthyroid state without medication. Indeed, the hyperthyroid state seen on the first examination in case 3 changed to euthyroid again after 2 months. These patients might also be considered to have euthyroid Graves' by a loose definition.

Transient and mild hyperthyroid states can be seen not only in patients with euthyroid Graves ophthalmopathy but also in patients who had suffered from persistent hyperthyroidism and lapsed into remission following prolonged medication (see case 5, Fig. 7). This case was judged to be in remission after 3 years of MMI treatment.
Two episodes of transient TSH suppression in association with an increase in serum $T_{4}$ and $T_{3}$ were observed after cessation of the antithyroid drug. During prodromal and postdromal stages of hyperthyroid Graves' disease, transient hyperthyroid states are likely to occur not so infrequently. The lack of a persistent increase in TBII may be a clue in discriminating these patients from those with persistent hyperthyroid Graves' disease. Whether all euthyroid patients with Graves' ophthalmopathy behave similarly, and the reason for not being able to continue to produce TBII, remain to be elucidated. We have recently observed that patients with euthyroid Graves' ophthalmopathy have unique associations with HLA which are quite different from those 'of hyperthyroid Graves' or even of Graves' ophthalmopathy (Inoue et al., unpublished observation). Such a genetic

Fig. 7

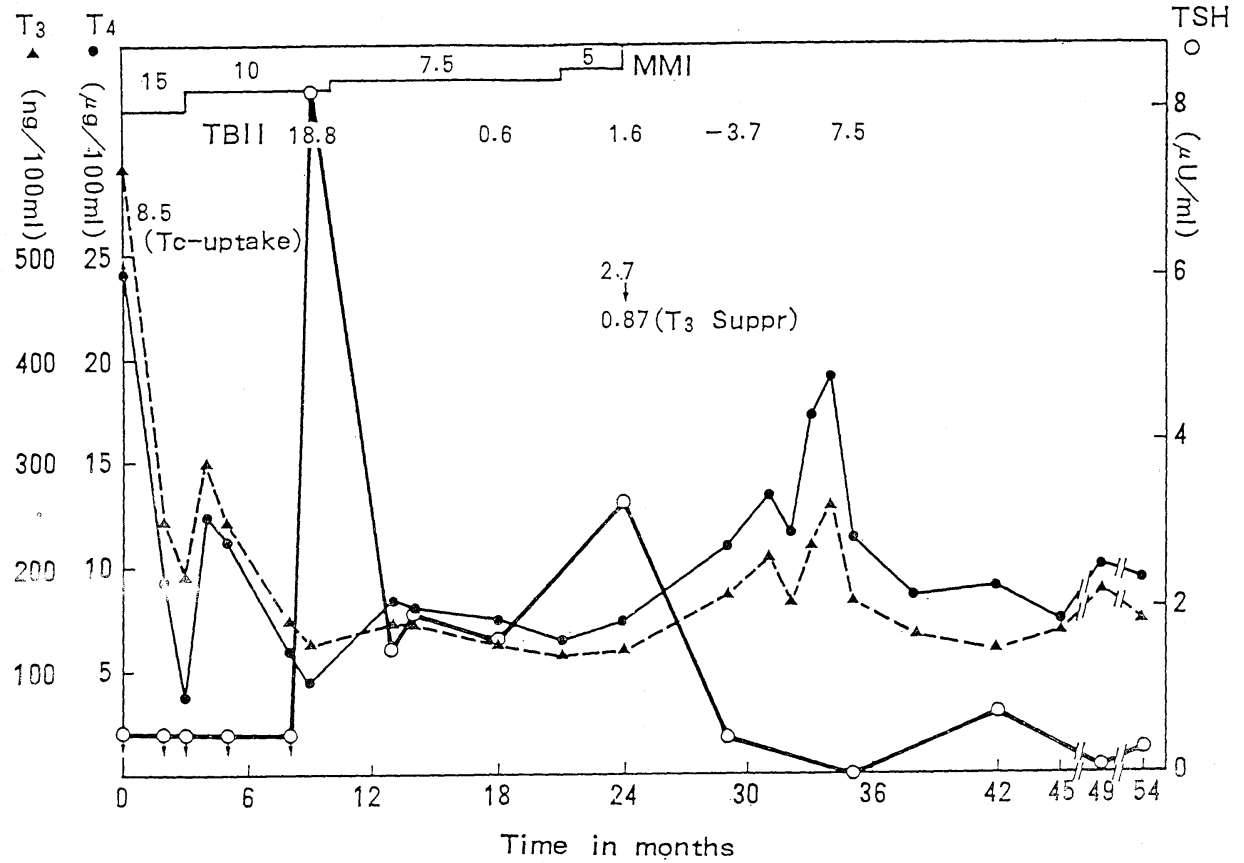

Fig. 7. Changes in thyroid functions and TBII in a Graves' patient (case 5). A 30-year-old female patient who had been treated with a high dose of MMI at another institution for more than 1 year. The clinical course demonstrated in the figure is that of after our initial observation. 
difference may be related to their unique characteristics.

\section{Acknowledgements}

The authors thank Miss Mie Katsu for excellent secretarial assistance and $\mathrm{Mr}$. Daniel Mrozek for reviewing and proofreading the manuscript.

A part of this study was supported by a grant-in-aid from the Japanese Ministry of Health and Welfare (uncontractile disease).

\section{References}

Akamizu, T., S. Bito, T. Ishihara, K. Sawada, K. Ikekubo and T. Mori (1984). Fundamental and Clinical examinations for TBI kit. Clin. Endocrinol. (Tokyo) 32, 195-204. (In Japanese). Akamizu, T., T. Mori, H. Ishii, T. Yokota, H. Nakamura and H. Imura (1987). Clinical significance of elevated labeled TSH binding (LTB) activity in sera of patients with Graves' disease and other thyroid disorders. J. Endocrinol. Invest. 10, 459-464.

Bidey, S. P., N. J. Marshall and R. P. Ekins (1983). Bioassay of thyroid-stimulating immunoglobulins using human thyroid cell cultures : optimization and clinical assessment. Clin. Endocrinol. $(O x f)$ 18, 193-206.

Etienne, J., L. D. Kohn and R. J. Wiland (1976). Studies on the mechanism of experimental exophthalmos. In: Thyroid Research (J. Robbins \& L. E. Braverman eds.), Elsevier, New York. pp. 380-382.

Gorman, C. A. (1978). The presentation and management of endocrine ophthalmopathy. Clin. Endocrinol. Metab. (London) 7, 67-96.

Kasagi, K., J. Konishi, K. Arai, T. Misaki, Y. Iida, K. Endo and K. Torizuka (1986). A sensitive and practical assay for thyroidstimulating antibodies using crude immunoglobulin fractions precipitated with polyethylene glycol. J. Clin. Endocrinol. Metab. 62. 855-862.

Kasagi, K., J. Konishi, Y. Iida, Y. Tokuda. K. Arai, K. Endo and K. Torizuka (1987). A sensitive and practical assay for thyroidstimulating antibodies using FRTL-5 thyroid cells. Acta Endocrinol. (Copehn) 115, 30-36.
Klee, G. G. and I. D. Hay (1987). Assessment of sensitive thyrotropin assays for an expanded role in thyroid function testing: proposed criteria for analytic performance and clinical utility. J. Clin. Endocrinol. Metab. 64, 461471.

Kohn, L. D. (1985). Thyrotropin receptor and thyroid autoimmune disease. Adv. Endocrinol. (Tokyo) 3, 159-181

Kosugi, S., T. Mori and H. Imura (1989). Mechanisms by which low salt condition increases sensitivity of thyroid stimulating antibody assay. Endocrinol. 125, 410-417.

Mori, T., H. Imura, S. Bito, K. Ikekubo, S. Inoue, S. Hashida, E. Ishika wa and H. Ogawa (1987). Clinical usefulness of a highly sensitive enzyme-immunoassay of TSH. Clin. Endocrinol. $(O x f)$ 27, 1-10.

Munro, R. E., L. Lamki, V. V. Row and R. Volpe (1973). Cell-mediated immunity in the exophthalmos of Graves' disease as demonstrated by the migration inhibition factor (MIF) test. J. Clin. Endocrinol. Metab. 37, 286-292.

Solomon, D. H., I. J. Chopra, U. Chopra and F. J. Smith (1977). Identification of subgroups of euthyroid Graves' ophtalmopathy. N. Eng. J. Mee. 296. 181-186.

Tamai, H., T. Nakagawa, N..Ohsako, O. Fukino, H. Takahashi, F. Matsuzuka, K. Kuma and S. Nagataki (1980). Changes in thyroid functions in patients with euthyroid Graves' disease. J. Clin. Endocrinol. Metab. 50, 108112.

Teng, C. S., B. R. Smith, B. Clayton, D. C. Evered, F. Clark and R. Hall (1977). Thyroidstimulating immunoglobulins in ophthalmic Gravess. Clin. Endocrinol. (Oxf) 6, 207-211.

Vitti, P., C. M. Rotella, W. A. Valente, J. Cohen, S. M. Alog, P. Laccetti, F. S. AmbesiImpiombato, E. F. Grollman, A. Pinchera, R. Toccafondi and L. D. Kohn (1983). Characterization of optimal stimulatory effects of Graves' monoclonal and serum Immunoglobulin G on adenosine 3' 5'-monophosphate production in FRTL-5 thyroid cells : a potential clinical assay. J. Clin. Endocrinol. Metab. 57, 782-791.

Zakarija, M., J. M. Mckenzie and K. Banovac (1980). Clinical significance of assay of thyroid-'disease timulating antibody in Graves' disease. Ann. Int. Med. 93, 28-32. 